\title{
Timing of anaphase-promoting complex activation in mouse oocytes is predicted by microtubule-kinetochore attachment but not by bivalent alignment or tension
}

\author{
Simon I. R. Lane, Yan Yun and Keith T. Jones*
}

\begin{abstract}
SUMMARY
Homologous chromosome segregation errors during meiosis I are common and generate aneuploid embryos. Here, we provide a reason for this susceptibility to mis-segregation by live cell imaging of mouse oocytes. Our results show that stable kinetochoremicrotubule attachments form in mid-prometaphase, 3-4 hours before anaphase. This coincided with the loss of Mad2 from kinetochores and with the start of anaphase-promoting complex/cyclosome (APC/C)-mediated cyclin B1 destruction. Therefore, the spindle assembly checkpoint (SAC) ceased to inhibit the APC/C from mid-prometaphase. This timing did not coincide with bivalent congression in one-third of all oocytes examined. Non-aligned bivalents were weakly positive for Mad2, under less tension than congressed bivalents and, by live-cell imaging, appeared to be in the process of establishing correct bi-orientation. The time from when the APC/C became active until anaphase onset was affected by the rate of loss of CDK1 activity, rather than by these non-aligned bivalents, which occasionally persisted until anaphase, resulting in homolog non-disjunction. We conclude that, in oocytes, a few erroneous attachments of bivalent kinetochores to microtubules do not generate a sufficient SAC 'wait anaphase' signal to inhibit the APC/C.
\end{abstract}

KEY WORDS: Cell cycle, Oocyte, Spindle assembly checkpoint, Mouse

\section{INTRODUCTION}

In mammals, the first meiotic division is hormonally triggered in the hours preceding ovulation, and results in the segregation of homologous chromosomes (bivalents). Sister chromatids, which remain attached in meiosis I, are then only segregated in meiosis II, a division therefore resembling mitosis. A notable feature of the meiotic segregation of bivalents in women is that it is a particularly error-prone event, leading to aneuploid embryos (Hassold and Hunt, 2009; Jones, 2008).

Aneuploidy is regarded as a driver of abnormal cell function and tumorigenicity, and dividing cells have multiple checkpoints that act to prevent chromosome mis-segregation (Holland and Cleveland, 2009; Williams and Amon, 2009). During mitosis, correct segregation is achieved through the mitotic checkpoint/spindle-assembly checkpoint (SAC), which can delay anaphase until amphitelic microtubule-kinetochore attachments are complete (Khodjakov and Pines, 2010; Musacchio and Salmon, 2007). Prior to this, the kinetochores produce a diffusible 'wait-anaphase' signal that sequesters Cdc20, an activator of the anaphase-promoting complex/cyclosome (APC/C), which is responsible for the degradation of key substrates at the metaphase-anaphase transition (Peters, 2006; $\mathrm{Yu}, 2007)$. This stabilizes the APC/C substrates cyclin B1 and securin, thereby maintaining CDK1 activity and inhibiting separase (Peters, 2006). The signal is regarded to be exquisitively sensitive, with a single unoccupied kinetochore enough to prevent anaphase (Rieder et al., 1995).

School of Biomedical Sciences and Pharmacy, University of Newcastle, Callaghan, NSW 2308, Australia.

*Author for correspondence (keith.jones@newcastle.edu.au)

Accepted 2 March 2012
There is currently much interest in what satisfies the SAC. In mitosis, microtubule attachment to kinetochores per se, the resulting tension across the kinetochore generated by stretching or the tension across the centromere of amphitelically attached sister kinetochores have all been suggested to nullify the signal that keeps the APC/C inactive (Khodjakov and Pines, 2010; Maresca and Salmon, 2010; Santaguida and Musacchio, 2009). It is still unclear whether the prevalence of aneuploidy in mammalian oocytes reflects a defect in the SAC during meiosis. Evidence that a univalent $\mathrm{X}$ chromosome can divide reductionally during meiosis $\mathrm{I}$ in mice suggested that mammalian oocytes might not possess a SAC (LeMaire-Adkins et al., 1997) but more recent studies have all shown mouse oocytes respond to SAC protein knockdown or loss as do somatic cells, with an increase in aneuploidy (Hached et al., 2011; Homer et al., 2005; Li et al., 2009; McGuinness et al., 2009; Wei et al., 2010; Yin et al., 2006). There is evidence in both mice and fission yeast that univalents in meiosis I could evade the SAC by establishing biorientation and so bypass the normal requirement to monoorientate (Kouznetsova et al., 2007; Sakuno et al., 2011). However, in a Mlh1 knockout mouse, in which many univalents are generated in meiosis I, these appear not to bi-orientate (Nagaoka et al., 2011), although they do generate a $\sim 2$ hour delay in polar body extrusion. It is not currently known whether under such circumstances the SAC becomes engaged but fails to fully arrest the oocyte, analogous to the process of 'mitotic slippage' in somatic cells (Brito and Rieder, 2006).

What remains to be investigated, therefore, are the factors that contribute to SAC silencing and APC/C activation during the normal course of female meiosis I. Here, we have focused in detail on the timing of the loss of the SAC protein Mad2 from kinetochores in mouse oocytes, providing through such observations, an account of why segregation errors arise in oocytes and how the timing of meiosis I is controlled. 


\section{MATERIALS AND METHODS}

\section{Materials}

All chemicals were from Sigma-Aldrich (Australia), unless stated otherwise. Stocks of nocodazole $(40 \mu \mathrm{M})$, roscovitine $(100 \mathrm{mM})$ and flavopiridol $(5 \mathrm{mM})$ were stored in DMSO and used at a minimum dilution of $0.1 \%$.

\section{Animals and oocyte culture}

F1 hybrid mice (C57B16 females $\times$ CBA males) were used in accordance with the Australian Code of Practice for the Care and Use of Animals for Scientific Purposes, and were approved by the University of Newcastle Animal Care and Ethics Committee. Oocytes were collected from mice injected with $10 \mathrm{IU}$ equine serum gonadotropin (Invitrogen, New Zealand) in M2 media as described previously (Reis et al., 2006). For longer term culture, oocytes were washed into MEM (Gibco) with $20 \% \mathrm{FCS}$ at $37^{\circ} \mathrm{C}$ in $5 \% \mathrm{CO}_{2}$ (Holt et al., 2011). Milrinone $(1 \mathrm{mM})$ was added to maintain prophase I arrest (Holt et al., 2010). For maturation studies, oocytes were synchronized by incubation in milrinone for 2 hours followed by its rapid washout. Only oocytes that underwent NEB 30-60 minutes after milrinone removal were used.

\section{cRNA manufacture}

cRNA was transcribed in vitro from purified, linear dsDNA template using a mMessage T7 RNA kit (Ambion) or a T3 RNA polymerase kit (Promega). cRNA was suspended in nuclease-free water and its concentration determined by photospectrometry or gel electrophoresis.

\section{Microinjection and live cell imaging}

cRNA microinjections were performed in M2 media on the stage of an inverted TE3000 Nikon microscope using a $37^{\circ} \mathrm{C}$ heated chamber and Narishige micromanipulators (Madgwick et al., 2006). Pressure injections to achieve a $0.2-3 \%$ oocyte volume were performed as described previously (Gorr et al., 2006). cRNAs were injected with pipette tip concentrations of $\sim 500 \mathrm{ng} / \mu \mathrm{l}$. Images were captured by epifluorescence using a Nikon Biostation IM $(20 \times$ objective) or a Nikon TE3000 microscope $(20 \times$ objective and Roper Scientific, UK, CCD camera), or by confocal scanning laser microscopy on an Olympus FV1000 $(60 \times$ objective).

\section{Immunofluorescence}

Oocytes were fixed in 2\% formaldehyde in PHEM buffer (60 mM Pipes, $25 \mathrm{mM}$ HEPES, $25 \mathrm{mM}$ EGTA, $4 \mathrm{mM} \mathrm{MgSO}_{4}$ ) containing $0.5 \%$ Triton $\mathrm{X}-100$ and $1 \mu \mathrm{M}$ taxol. For analysis of k-fibers, some oocytes were incubated in pre-cooled M2 media on ice for 10 minutes before fixation or treated with a high $\mathrm{Ca}^{2+}$ permeabilizing buffer for 2 minutes $[100 \mathrm{mM}$ Pipes (pH 7.0), $1 \mathrm{mM} \mathrm{MgCl} 2,0.1 \mathrm{mM} \mathrm{CaCl}_{2}, 0.1 \%$ Triton X-100]. Oocytes were then fixed, washed and further permeabilized in PBS containing $0.5 \%$ Triton X100, then blocked in 7\% normal goat serum with PBS-Tween. Immunofluorescence was performed using antibodies for tubulin (1:400, A11126, Invitrogen, Australia), CREST (1:400, 90CCS1058, Bioclone Australia) and Mad2 (1:1000, a kind gift from Dr R. H. Chen, Taipei, Taiwan). Antibody solutions were supplemented with $7 \%$ goat serum and $0.2 \%$ Tween 20 , and incubated overnight at $4{ }^{\circ} \mathrm{C}$. Secondary antibodies were Alexa 633-, 555- and 488-conjugated (Invitrogen). Oocytes were briefly stained with Hoechst $(20 \mathrm{mg} / \mathrm{ml})$ before mounting on glass slides with Citifluor (Citifluor, UK). In Mad2/CREST fluorescence experiments or stable k-fiber experiments, different groups of oocytes were processed for immunofluorescence in parallel using the same antibody solutions.

\section{Immunoblotting}

Oocytes were briefly washed in PBS with $1 \%$ PVP, suspended in $1 \times$ sample buffer (Invitrogen) and run on a NuPage 10\% gel (Invitrogen) according to the manufacturer's instructions. Immunoblotting (50 oocytes per lane) was performed using antibodies against securin $(1: 200$, ab3305, Abcam, UK), cyclin B1 (1:500, ab72, Abcam), and actin (1:400, ab3280, Abcam) overnight at $4{ }^{\circ} \mathrm{C}$, using $3 \%$ BSA in PBST. ECL Plus (GE Healthcare, UK) was used for detection with an anti-mouse $\operatorname{IgG}(1: 5000$, P0447, DAKO, UK).

\section{Confocal imaging and image analysis}

An Olympus FV1000 fitted with a $60 \times$ objective was used on fixed cells for all confocal microscopy. $Z$-stacks were compiled with $0.5 \mu \mathrm{m}$ intervals. For Mad2/ CREST or k-fiber analysis, scans were performed using identical settings and on the same day.

All images were analyzed using Metamorph and Metafluor software (Universal Imaging, PA, USA) or ImageJ (NIH, Bethesda, USA) and figures assembled with Adobe Illustrator CS4. Calculation of Mad2-YFP fluorescence at kinetochores was made by recording mean fluorescence intensity from a $1 \mu \mathrm{m}$ diameter circle centered on the YFP signal associated with bivalents. This intensity reading was self-ratioed with respect to the start of the recording (3 hours post NEB). Calculation of Mad2 immunostaining at kinetochores was made by recording the mean fluorescence intensity of both Mad2 and CREST colocalized immunosignals, which were subtracted for background.

\section{Statistical analysis}

$P$ values were calculated using an unpaired Student's $t$-test or by ANOVA, with Tukey's post-hoc analysis (Minitab16, PA, USA).

\section{RESULTS \\ Timing of k-fiber formation and APC/C activation in mouse oocytes}

Prophase I oocytes in dictyate arrest spontaneously undergo nuclear envelope breakdown (NEB) when disassociated from the ovary. The extrusion of the first polar body, which is an event that marks the completion of meiosis I, occurred at $8.1 \pm 0.7$ hours (mean \pm s.d., $n=100$ ) post-NEB in the mouse strain used here. Cyclin B1 and securin degradation, however, began a few hours earlier, at $\sim 5$ hours post-NEB (supplementary material Fig. S1A-C). This same timing, at $7.9 \pm 0.4$ hours, $(n=26$; supplementary material Fig. S1D,E) was also observed with exogenous cyclin B1, following 1.0 pg cRNA cyclin B1-GFP injection. This procedure generates 25$50 \mathrm{ng}$ of protein hour $^{-1}$ and approximates to $5-10 \%$ of endogenous cyclin B1 concentration (Madgwick et al., 2004).

These initial observations show that cyclin B1 and securin degradation begin about 3 hours before either anaphase or cytokinesis (polar body extrusion), which are parallel events in mouse oocytes (Verlhac et al., 2000). By comparison, the duration of cyclin B1 degradation in somatic cells is about 20 minutes (Clute and Pines, 1999; Gavet and Pines, 2010). Given that in both oocytes and somatic cells this APC/C substrate loss is Cdc20 dependent (Jin et al., 2010; Li et al., 2007; Reis et al., 2007), the data collectively point towards a period of meiotic APC/C activity that lasts 10 times longer, from $\mathrm{APC} / \mathrm{C}^{\mathrm{cdc} 20}$ activation to cytokinesis, than it does in mitosis of most cultured cell lines.

To place this prolonged prometaphase period of cyclin B1 and securin degradation in context with major meiotic events, we first examined the integrity of the spindle at the time of $\mathrm{APC} / \mathrm{C}$ activation. In mouse oocytes, lateral attachment of microtubules to kinetochores precedes more stable end-on k-fiber formation (Brunet et al., 1999), as it does in mitosis. End-on k-fibers, which can be assessed in cells following either cold-shock or calcium treatment (Amaro et al., 2010; DeLuca et al., 2006; Kitajima et al., 2011; Salmon and Segall, 1980; Toso et al., 2009; Weisenberg and Deery, 1981), were observed on the vast majority of kinetochores at 5 hours, but not 4 hours, post-NEB. This observation was made using either $\mathrm{Ca}^{2+}$ treatment or cold shock (Fig. 1A; supplementary material Fig. S2A,B), and stable k-fibers continued to develop during the remaining 3 hours of meiosis I, as assessed by coldshock (supplementary material Fig. S2A).

Monotelic attachment of sister kinetochores ensures that homologous chromosomes rather than sister chromatids separate in meiosis I. At 5 hours post-NEB we could perform individual 


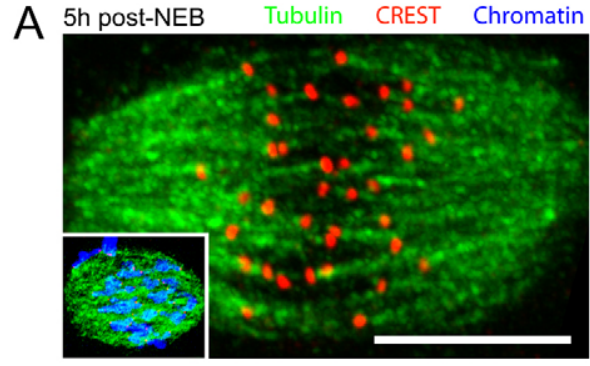

4h post-NEB

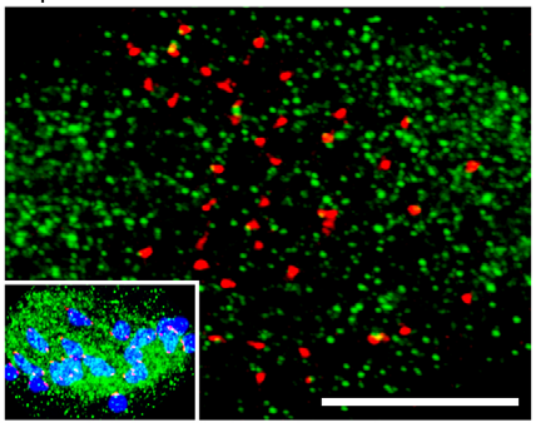

B

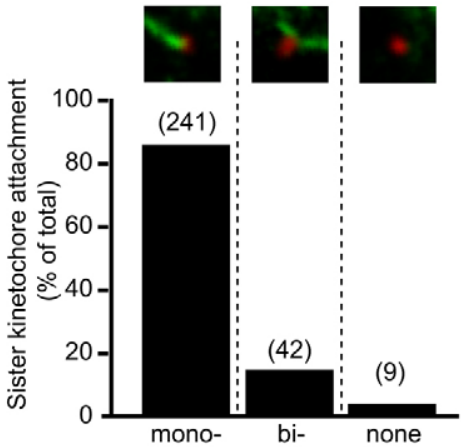

Fig. 1. Kinetochore occupancy by microtubule k-fibers at $\mathbf{5}$ hours after NEB. (A) Representative meiotic spindles from $\mathrm{Ca}^{2+}$-treated oocytes at 4 hours ( $n=6$ oocytes) and 5 hours ( $n=29$ oocytes) after NEB. Insets, oocytes fixed at the same timepoint but under conditions that immunostain for all tubulin, not just k-fibers. Scale bars: $10 \mu \mathrm{m}$. (B) Sister kinetochore attachment status to k-fiber microtubules (mono-, monooriented; bi-, bi-oriented) at 5 hours after NEB. The number of sister kinetochores examined are given in parentheses.

analysis on sister kinetochore pairs to determine their attachment status to k-fibers. In summary, over $90 \%$ ( $n=292 / 320$, from eight oocytes) of sister kinetochore pairs were sufficiently resolved by confocal analysis to allow for accurate assessment. Of those sister kinetochore pairs that could be resolved, 97\% $(n=283 / 292)$ were associated with k-fibers (Fig. 1B), with a large majority of these $(>80 \%)$ having monotelic attachment. These data suggest that when the $\mathrm{APC} / \mathrm{C}$ commences its activation, the majority of sister kinetochore pairs have established end-on interactions with microtubules, although such attachment is not necessarily correct.

\section{Mad2 loss from kinetochores coincides with APC/C activation}

Given the seemingly synchronous appearance of stable k-fibers and initiation of APC/C activation by 5 hours post-NEB, we wanted to determine whether the $\mathrm{SAC}$ is also satisfied at this time. To assess SAC activity, live oocytes expressing H2B-mCherry and Mad2-YFP were imaged by $4 \mathrm{D}$ confocal microscopy during meiosis I. At 3-4
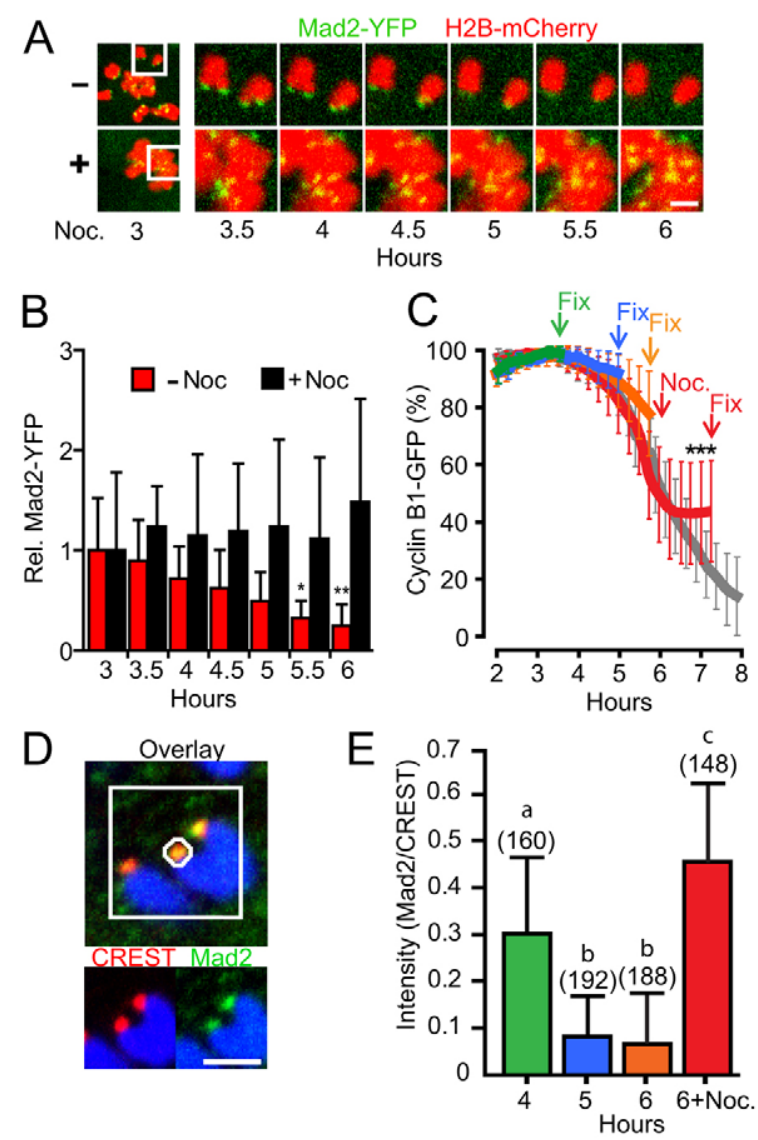

Fig. 2. Similar timing for loss of Mad2 from kinetochores and initiation of cyclin B1 degradation. (A) Representative timelapse of Mad2-YFP and H2B-mCherry fluorescence in live oocytes, with or without nocodazole ( $n=5$ oocytes); insets at 3 hours are enlarged in subsequent timepoints. (B) Kinetochore Mad2-YFP intensity ratio, expressed relative to 3 hours, at times post-NEB with or without nocodazole ( $n=10-16$ kinetochores per timepoint). ${ }^{*} P<0.05,{ }^{*} * P<0.01$ (ANOVA, Tukey's) compared with the 3-hour timepoint. (C) Mean cyclin B1-GFP fluorescence levels in groups of oocytes, expressed as a percentage of maximum reached, which were fixed (except gray trace) or treated with nocodazole at the times indicated. ${ }^{*} P<0.05$ compared with control (gray). (D) Mad2 and CREST immunostaining of a representative bivalent of an oocyte at 4 hours post NEB. Inner circle, area of Mad2 and CREST fluorescence intensity measured following a background subtraction made in the outer square. (E) Mad2/CREST ratios, made on the oocyte groups from $C$, using the technique of $D$; groups with different letters $(a, b, c)$ are significantly different from one another $(P<0.001)$; the number of kinetochores analyzed are in parentheses. Scale bars: $2 \mu \mathrm{m}$ in A,D. All error bars are s.d.

hours following NEB, Mad2-YFP was detected on all kinetochores, consistent with the observed lack of end-on microtubule attachment to kinetochores at this time (supplementary material Fig. S2) and the idea that this lack of attachment would lead to SAC activity (Kim et al., 2010; Kulukian et al., 2009). A significant drop in kinetochore Mad2-YFP was then observed during the following 2-3 hours, a process that was not due to photobleaching because the YFP signal was maintained for several hours when the experiment was repeated in the presence of nocodazole to destabilize microtubules (Fig. 2A,B).

We confirmed that there was a loss of endogenous Mad2 from kinetochores, coincident with $\mathrm{APC} / \mathrm{C}$ activation, using oocytes injected with cyclin B1-GFP cRNA. Oocytes were fixed prior to 
$\mathrm{APC} / \mathrm{C}$ activation at 4 hours post-NEB (Fig. 2C, green) or after $\mathrm{APC} / \mathrm{C}$ activation at 5 and 6 hours (Fig. 2C, blue and orange, respectively). Some oocytes at 6 hours were also incubated with nocodazole for 1 hour before fixation so as to inhibit the $\mathrm{APC} / \mathrm{C}$ (Fig. 2C, red). All fixed cells were then immunolabeled for Mad2 and CREST, and the respective fluorescent signals on kinetochores were ratioed for each group (Fig. 2D). Kinetochores from oocytes fixed after the onset of $\mathrm{APC} / \mathrm{C}$ activation were found to have significantly less associated Mad2 when compared with those fixed before cyclin B1 degradation onset or treated with nocodazole. Therefore, we could quantitate a significant loss in kinetochoreassociated Mad2 between 4 and 5 hours post-NEB, coinciding with the timing of stable microtubule-kinetochore attachment and APC/C activation (Fig. 2E). We also confirmed that the same timing of loss of Mad2 from kinetochores was observed in the absence of exogenous cyclin B1, using fixed oocytes at specific timepoints during meiosis I (supplementary material Fig. S3).

\section{APC/C activation is not associated with bivalent congression}

The above data indicated that the overall level of SAC-induced $\mathrm{APC} / \mathrm{C}$ inhibition was diminished at about 3 hours before anaphase onset and cytokinesis, as judged by the initiation of cyclin B1 degradation and the loss of Mad2 from kinetochores. Therefore, we wanted to investigate the extent to which bivalent congression was complete at this time. Maturing oocytes were monitored for $\mathrm{APC} / \mathrm{C}$ activity by waiting for a steady loss in cyclin B1-Venus fluorescence following its cRNA injection at the prophase I stage. When such a decline was observed, further cyclin B1 imaging was stopped and the status of congression assessed using a Hoechst dye. Interestingly, in nearly half of all the oocytes examined $(43 \%, n=42)$ we could readily observe chromosomes that were not positioned near the spindle equator (Fig. 3). These were confirmed as bivalents, rather than univalents, which may have been generated by premature reductional division of the bivalent (supplementary material Fig. S4).

\section{Many non-aligned bivalents have attachment defects and can recruit Mad2}

From the above it seems likely that once the APC/C has become active in prometaphase then the SAC is not sufficiently re-engaged to inhibit meiotic progression during the process of establishing
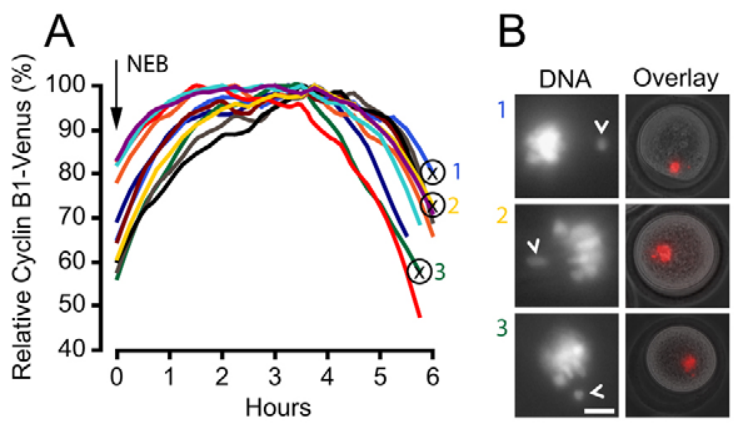

Fig. 3. Cyclin B1 degradation in the presence of non-aligned bivalents. (A) Representative cyclin B1-Venus levels in ten maturing oocytes ( $n=42)$, expressed as a percentage of the maximum

fluorescence. Time 0 hours corresponds to NEB as indicated, and at the end of the recording oocytes were imaged for chromatin. This event is marked (cross inside a circle) for the three oocytes shown in B.

(B) Chromatin and bright-field images of three numbered oocytes from $\mathrm{A}$, at the times indicated. Arrowheads indicate non-aligned bivalents. Scale bar: $5 \mu \mathrm{m}$. bivalent bi-orientation. We decided to examine this by assessing Mad2 localization to the kinetochores of all bivalents at 5 hours post-NEB, a time when the $\mathrm{APC} / \mathrm{C}$ is active in degrading cyclin B1. The bivalents from fixed oocytes, immunostained for Mad2, CREST and labeled for chromatin, were individually assessed for congression, as measured by the distance of their kinetochores from the spindle equator. Those bivalents having one or both sister kinetochores pairs within $4 \mu \mathrm{m}$ of the spindle equator were considered to be congressed, and those with both kinetochores outside this distance were considered to be non-aligned (Fig. 4A). This distance from the spindle midzone meant that by our definition non-aligned bivalents were always clearly separated from all the other bivalents that had congressed. The mean distance of kinetochores from non-aligned bivalents from the spindle equator was $6.3 \pm 1.3 \mu \mathrm{m}(n=52)$.
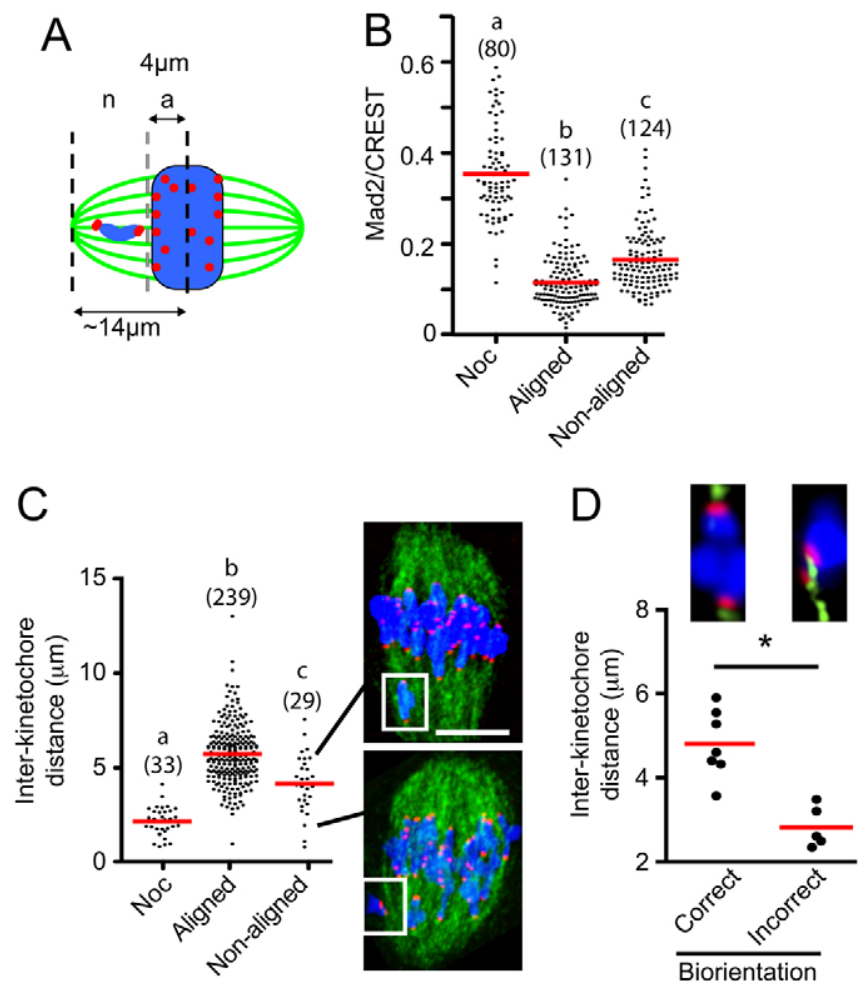

Fig. 4. Non-aligned bivalents have more Mad2 and are under less tension than those on the spindle equator. (A) Meiotic spindle schematic, with bivalents classified as aligned ' $a$ ', with kinetochores $\leq 4 \mu \mathrm{m}$ from the spindle midzone, or non-aligned ' $n$ ' (>4 $\mu \mathrm{m})$. (B) Mad2/CREST ratios in bivalents at 5 hours post-NEB that were either aligned or non-aligned, or following nocodazole addition. (C) Interkinetochore distances of bivalents that are aligned, non-aligned or from nocodazole-treated oocytes. Inter-kinetochore distance is defined as the separation of the two sister kinetochore pairs within a bivalent. Two examples of meiotic spindles are shown with a non-aligned bivalent and its corresponding inter-kinetochore distance (white box, line). Scale bar: $10 \mu \mathrm{m}$. (D) Inter-kinetochore distance in non-aligned bivalents ( $n=12)$. Bivalents were categorized as being bi-oriented $(n=7)$, with the two pairs of sister kinetcohores being attached to opposite spindle poles, or non-bi-oriented $(n=5)$. The non-bi-oriented bivalent shown has both sister pairs attached to the same pole. (B-

D) Parentheses indicate number of kinetochores examined. (A,C,D) Chromatin is shown in blue; tubulin, green; kinetochores, red. (BD) Groups with different letters $(a, b, c)$ or marked by an asterisk are significantly different from one another $(P<0.001)$; mean values are indicated by horizontal red lines. 
We observed that Mad2 staining on kinetochores of non-aligned bivalents was significantly more intense than on those that were congressed (Fig. 4B, $P<0.001$, measurements on both pairs of sister kinetochores were used). However, the amount of Mad2 on these non-aligned bivalents remained significantly below the levels achieved following complete loss of microtubule attachment through nocodazole addition (Fig. 4B) and also below the Mad2 levels on kinetochores at 4 hours post-NEB (supplementary material Fig. S3), at which time no k-fibers can be observed (supplementary material Fig. S2).

The above data suggest that many non-aligned bivalents were associated with raised levels of Mad2, compared with bivalents at the spindle equator. If the attachment of non-aligned bivalents were correct, we would predict that microtubule stretch would develop tension across them, leading to greater separation of their two sister kinetochore pairs. For bivalents at or near the spindle equator and so classified as aligned, the mean separation of the two pairs of sister kinetochores was 5.7 $\pm 1.6 \mu \mathrm{m}$ ( \pm s.d., $n=239$ pairs; Fig. $4 \mathrm{C})$, significantly greater than that measured in non-aligned bivalents (4.2 $\pm 1.6 \mu \mathrm{m} ; n=29 ; P<0.001$; Fig. $4 \mathrm{C})$. Although mean tension was weaker in non-aligned bivalents, it was not absent, given that nocodazole added to collapse all tension across the bivalents significantly reduced kinetochore separation still further (Fig. 4C).

To examine the k-fiber attachment of the two sister kinetochore pairs associated with each non-aligned bivalent, oocytes were immunostained for tubulin and CREST following $\mathrm{Ca}^{2+}$ treatment at 5 hours post-NEB. In total, 12 non-aligned bivalents were observed, and in just over half $(58 \%, n=7 / 12)$, the bivalent was observed to be bi-oriented with monotelic attachment of each sister pair to opposite poles. In the remaining bivalents $(42 \%, n=5 / 12)$ the attachment was incorrect, with either monotelic attachment of both sister pairs to the same pole $(n=1)$, one kinetochore pair attaching to both poles $(n=1)$, one kinetochore pair being vacant $(n=1)$ or a combination of the above $(n=2)$. As expected, the non-aligned bivalents that were classified as bi-oriented by k-fiber attachment and orientation had much greater stretch across them than those bivalents that were non-bi-oriented (Fig. 4D).

In summary, individual non-aligned bivalents can have characteristics that overlap with bivalents under tension on the spindle equator and those in which all microtubule attachment has been abolished with nocodazole. Their kinetochores have an intermediate level of Mad2 association and appear to be under less tension. Such observations are consistent with the non-aligned bivalents falling into one of two distinct categories. First, those that have failed to make correct attachments to microtubules at both spindle poles, a situation that generates unbalanced forces and bivalent movement towards the pole with greater attachment, thus generating the non-alignment in the first instance. Second, those non-aligned bivalents that have had their erroneous attachment corrected, becoming bi-oriented and as such are moving back towards the spindle equator. However, although this is the simplest interpretation of the above, and is consistent with recent reports tracking bivalent movements and error correction in meiosis I (Kitajima et al., 2011), here they are based on fixed oocytes only, and need further analysis in live cells.

\section{Non-aligned bivalents do not inhibit the APC/C and can generate aneuploidy}

So far, we had observed non-aligned bivalents in fixed oocytes. In order to understand more fully their provenance and fate, we expressed histone2B-mCherry (H2B-Cherry) and followed bivalent movements with high-temporal acquisition by $2 \mathrm{D}$ epifluorescence.
All oocytes examined displayed rapid oscillations of bivalents about the spindle midzone that were estimated to last 1-3 minutes but which result in only very small displacements from the spindle equator (supplementary material Movie 1, left panel). They appear to be analogous, in terms of duration and displacement, to the oscillations of sister chromatids during mitosis that have been reported elsewhere (Jaqaman et al., 2010; Skibbens et al., 1993). However, in addition, many of the oocytes had non-aligned bivalents, which were readily identifiable as separate from the spindle equator and which persisted over a much longer timeframe, from tens of minutes to hours (Fig. 5A; supplementary material Movie 1, right panel).

We wondered whether the presence of non-aligned bivalents had any effect on the timing of anaphase. If non-aligned bivalents were able to generate a robust SAC checkpoint and so inhibit the $\mathrm{APC} / \mathrm{C}$, then one would predict that this timing would be delayed, in the same way observed with low doses of the spindle poison nocodozale (Wassmann et al., 2003). Comparing oocytes in which bivalents remained aligned from the period 4.5 hours post NEB onwards with those where non-aligned bivalents were evident for at least 1 hour, we observed no difference in the timing of polar body extrusion (Fig. 5A,B). The lack of any change in the timing of anaphase in those oocytes with persistently non-aligned bivalents suggests they have no influence on SAC activity. However, if this were so, we should not observe any difference in $\mathrm{APC} / \mathrm{C}$ activity between the two groups. To test this, we measured the rate of cyclin B1-Venus degradation in oocytes co-expressing H2B-Cherry. As carried out previously, comparing oocytes in which bivalents remained aligned from the period 4.5 hours post NEB onwards with those where non-aligned bivalents were evident for at least 1 hour, we observed no difference in cyclin B1 degradation, measured by when it starts and stops, or by its maximal rate of loss (Fig. 5C,D).

We thought it important to examine the fate of non-aligned bivalent until the time of anaphase in oocytes co-expressing cyclin B1-GFP with H2B-Cherry. We observed that in $~ 70 \%$ of oocytes $(n=52)$, bivalents were congressed at the time of APC/C-induced cyclin B1 loss and maintained this congression until anaphase (supplementary material Movie 2), but not in $30 \%$, where nonaligned bivalents were observed to persist for extended times (Fig. 5E,F; supplementary material Movie 3). Therefore, we surmise that most of the non-aligned bivalents observed previously in fixed oocytes at 5 hours post-NEB (Fig. 4) are likely to be chromosomes that have not yet congressed at the spindle equator. However, at later meiotic times it was still possible to observe bivalents leave the metaphase plate, which would have been classified as congressed at earlier timepoints (Fig. 5E, arrowhead). Importantly, for the oocyte in the prevention of non-disjunction, the majority of these non-aligned bivalents did eventually congress at the metaphase plate in the time period before anaphase (Fig. 5F). However, in 2 out of the 51 oocytes examined (4\%), bivalents persisted until anaphase onset and underwent non-disjunction (Fig. 5E; supplementary material Movie 4).

Finally, we examined the movement of the kinetochores of nonaligned bivalents in live oocytes $(n=9)$ by injecting $\mathrm{GV}$ oocytes with CenpC-GFP and H2B-mCherry cRNA to confirm that nonaligned bivalents do indeed congress and become stably biorientated on the metaphase I spindle. Once bi-orientated, the bivalents moved towards the spindle equator and showed the same characteristics as all the other bivalents (supplementary material Movie 5). These live cell observations confirm the behavior interpreted from the fixed oocytes, in which non-aligned bivalents 

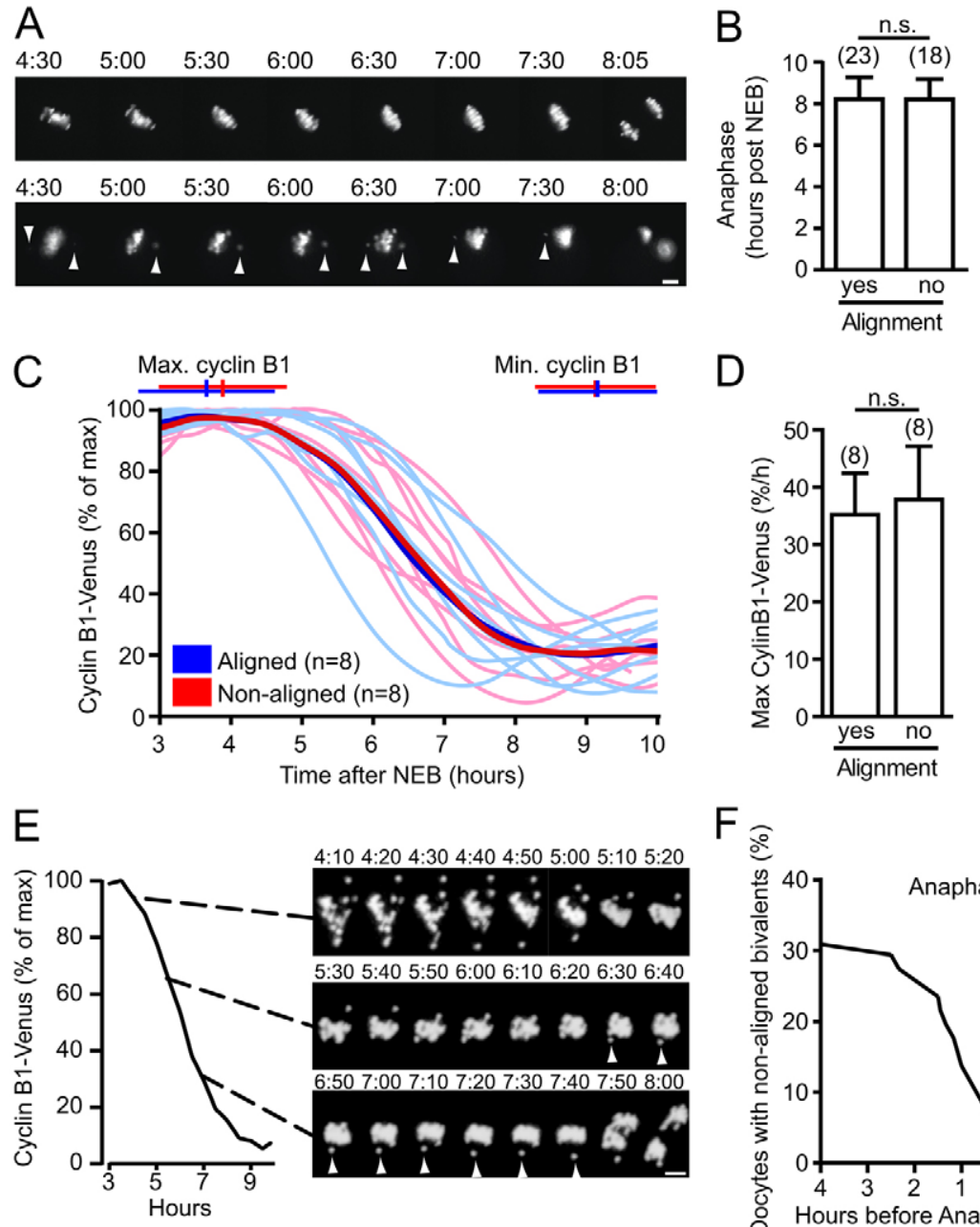

\section{$\mathrm{F}$}

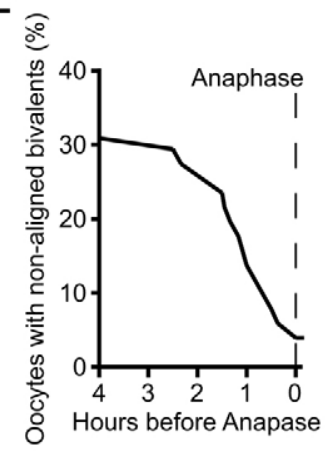

Fig. 5. Non-aligned bivalents fail to inhibit the APC/C and can undergo non-disjunction at anaphase. (A) Representative images of oocytes expressing $\mathrm{H} 2 \mathrm{~B}-\mathrm{mCherry}$, with either aligned bivalents present for the entire period of imaging (top) or nonaligned bivalents (bottom). (B) Timing of anaphase onset in oocytes with or without aligned bivalents. (C,D) Cyclin B1-Venus levels in oocytes with or without aligned bivalents, showing the time at which maximum and minimum cyclin $B 1$ levels were reached. Alignment was observed by co-expression of $\mathrm{H} 2 \mathrm{~B}-\mathrm{mCherry}$, and the means of individual recordings are in bold (D). Mean maximal rate of cyclin B1 degradation in those oocytes from C. (E) Cyclin B1Venus fluorescence and corresponding H2B-mCherry images, in an oocyte in meiosis I at the times indicated after NEB. Arrowheads indicate a non-aligned bivalent undergoing non-disjunction. Scale bar: $10 \mu \mathrm{m}$. (F) Timing of bivalent congression in oocytes measured against the time to anaphase-onset $(n=78)$. (A-D) Persistent non-aligned bivalents were classified as being present for periods of 1 hour or greater. $(B, D)$ Errors are s.d.; n.S., non-significant ( $P>0.05, t$ test); numbers of oocytes analyzed are in parenthesis. initially appear to be under less tension and move polewards, probably towards the pole with which it has established greater attachment. Correction of the erroneous attachment is then likely to occur, based on the observations that very few non-aligned bivalents do undergo non-disjunction, and the bi-oriented bivalent now under tension from both poles moves to the spindle equator.

\section{CDK1 activity is important in the timing of anaphase following APC/C activation}

What then dictates the timing of bivalent segregation in oocytes, given the $\mathrm{APC} / \mathrm{C}$ is activated 3-4 hours ahead of anaphase? One possibility is that the gradual decline of cyclin B1 over 3-4 hours maintains a high CDK1 activity and this lengthy period permits sufficient time for bi-orientation of all bivalents. To examine this, the pan-CDK inhibitor roscovitine (Bain et al., 2007) was used at $100 \mu \mathrm{M}$, a minimally effective dose to inhibit CDK1 in mouse oocytes, as judged both by its ability to block NEB and through in vitro CDK1 kinase assays (supplementary material Fig. S5) (Deng and Shen, 2000).

Roscovitine was added to maturing oocytes expressing H2BmCherry between 3 and 7 hours post-NEB, and the timing of anaphase recorded by timelapse imaging (Fig. 6A-C). The overall percentage of oocytes stimulated to undergo anaphase by $\mathrm{Cdk}$ inhibition increased as meiosis progressed. Thus, following roscovitine addition at 5 hours post-NEB, $87 \%$ of oocytes underwent anaphase, compared with fewer than $20 \%$ at 3 hours
(Fig. 6B). The ability of a few oocytes to show anaphase movement in the absence of $\mathrm{k}$-fibers at 3 hours would be consistent with the findings that at least in oocytes this anaphase pulling force can be provided by microtubules not associated with the kinetochore (Deng et al., 2009; Dumont et al., 2010). The large increase in the ability of oocytes to undergo anaphase when roscovitine is added between 3 and 4 hours (19.2 versus $71.4 \%$, $P<0.001)$ correlates well with the formation of stable microtubules between 4 and 5 hours after NEB, suggesting that this process is more efficient in the presence of $\mathrm{k}$-fibers.

Anaphase onset occurred prematurely following roscovitine addition at all times tested, therefore decreased CDK1 activity may be an important step in the timing of meiotic exit (Fig. 6A,C; supplementary material Movie 6). At 7 hours post-NEB, the most meiotically advanced time assessed, anaphase was $\sim 20$ minutes after drug addition (Fig. 6C). However, at earlier meiotic times anaphase onset was much more delayed, up to 55 minutes. This same anaphase timing was also observed using flavopiridol at a dose of $5 \mu \mathrm{M}$ (supplementary material Fig. S5) (Potapova et al., 2006), suggesting the delay is not specific to one CDK inhibitor. Instead, this delay is probably due to the need to recruit other pathways, such as those involving phosphatases, which are known to be needed for mitotic and meiotic exit (Schindler and Schultz, 2009; Schmitz et al., 2010). It was possible to restore the delay to anaphase after roscovitine addition in oocytes injected with a very high dose of cyclin B1-GFP cRNA (100 pg) 100-fold greater than 


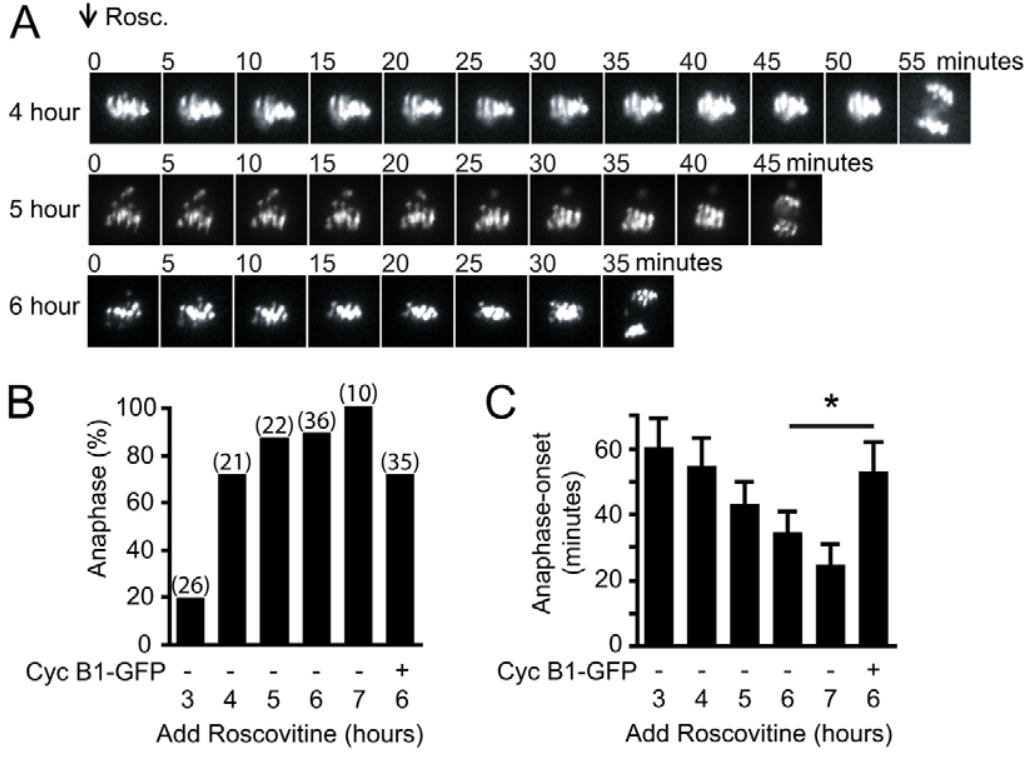

Fig. 6. Reduced CDK1 activity accelerates anaphase onset. (A) Representative H2B-mCherry fluorescence images every 5 minutes following $100 \mu \mathrm{M}$ roscovitine addition to oocytes at the times indicated post-NEB. (B,C) Percentage of oocytes undergoing anaphase (B) and associated timing of anaphase onset measured in minutes after roscovitine addition (C), calculated from timelapses in $\mathrm{A}_{;}$' ${ }^{\prime}$ ', oocyte injected with $100 \mathrm{ng}$ cyclin B1-GFP cRNA before NEB. (B) Numbers of oocytes analyzed in parentheses are the same for $C$. (C) ${ }^{*} P<0.001$, error bars are s.d. used previously, and which masks any observable cyclin B1-GFP degradation (not shown) and would be expected to give a high level of CDK1 activity (Fig. 6C).

\section{DISCUSSION}

In mitosis, complete congression of all sister chromatids onto a metaphase plate is closely coupled with the initiation of cyclin B1 degradation (Clute and Pines, 1999). Even in chromosomally unstable cell lines, anaphase is not observed without first achieving full alignment (Thompson and Compton, 2008). The observations here were therefore surprising, that the initiation of APC/C activity, measured by cyclin B1 degradation, in at least one-third of all mouse oocytes occurred several hours before complete bivalent congression. Instead, we found that the cyclin B1 loss was more closely associated with the attachment of the vast majority of sister kinetochore pairs to microtubules. Strain differences do influence the timing of meiosis I and cyclin B1 degradation in mice (Ledan et al., 2001; Polanski et al., 1998), but in our F1 hybrids, 5 hours post-NEB was when loss of this APC/C substrate was first seen and when $\sim 97 \%$ of all kinetochores were associated with $\mathrm{k}$-fibers.

At the kinetochores, the attachment of microtubules 5 hours post-NEB led to the loss of the SAC protein Mad2. It is likely that this event in meiosis, as in mitosis, is responsible for $\mathrm{APC} / \mathrm{C}$ activation. Unoccupied kinetochores are well known to recruit cytosolic Mad2, which is then thought to undergo conformational changes rendering it able to inhibit Cdc20 (De Antoni et al., 2005; Kim et al., 2010; Yu, 2006). It is not entirely clear how microtubule attachment causes $\mathrm{Mad} 2$ to be lost from kinetochores and the checkpoint silenced, but it does involve at least in part dynein motor-driven movement to the spindle poles and the Mad2-binding protein p31 comet (Gassmann et al., 2010; Griffis et al., 2007; Yang et al., 2007).

The reason why congression of sister chromatids in mitosis but not bivalents in meiosis is an event coupled with $\mathrm{APC} / \mathrm{C}$ activation may well be a reflection of the fact that building a spindle in the absence of centrosomes is less efficient and so takes longer to achieve (Hornick et al., 2011; Mahoney et al., 2006). Alternatively, it may be that achieving bi-orientation of bivalents through the monotelic attachment of sister kinetochore pairs is less efficient than the equivalent process with respect to sister chromatid bi- orientation in mitosis. However, independent of the reasoning for the delay in meiosis, in both instances kinetochore occupancy appears to be the major driver of $\mathrm{APC} / \mathrm{C}$ activation. The observation that many bivalents are not congressed at the spindle equator, and that these bivalents have much shorter inter-sister kinetochore distances, suggest that they are not fully bi-oriented and under maximal tension. Such bi-orientation is achieved in meiosis I by monopolar attachment of each sister kinetochore pair to opposite spindle poles, and this ensures maximal tension across the bivalent.

The present data are therefore consistent with a model in which initial attachment of microtubules to sister kinetochores satisfies the SAC, as also suggested in mitosis (Khodjakov and Pines, 2010), but that these attachments may be erroneous and need to be repaired. Indeed, this repair may be very common in mouse oocytes, given that three rounds of microtubule error correction have been calculated to occur for each bivalent, measured from the time of NEB until polar body extrusion (Kitajima et al., 2011). It is likely, but remains to be firmly established, that Aurora kinases play a part in this process, as they do in mitosis, by destabilizing erroneous attachment that fail to generate adequate tension across the kinetochores (Lane et al., 2010; Liu et al., 2009; Welburn et al., 2010; Yang et al., 2010).

In mouse oocytes, the time following SAC satisfaction corresponded to a long, 3- to 4-hour, period of cyclin B1 degradation, a length that has been observed previously in other studies (Herbert et al., 2003; Homer et al., 2005; McGuinness et al., 2009). This timing, of anaphase-onset from the start of APC/C activation, is found here to be shortened by CDK1 inhibition, suggesting at least in part that the period is governed by CDK1 activity. Consistent with this, mouse oocytes appear to contain much greater amounts of cyclin B1 than cultured somatic cells, so much so that it is in a several-fold molar excess to its kinase binding partner CDK1 (Arooz et al., 2000; Kanatsu-Shinohara et al., 2000).

The non-aligned bivalents that persist at this time had Mad2 associated with their kinetochores, but levels were much weaker when compared with those kinetochores that had not established any microtubule attachment or when microtubules were stripped off kinetochores by nocodazole. A weak Mad2 association with 
non-aligned bivalents would be in keeping with the finding that, in the majority of cases, the sister kinetochores have some attachment to microtubules, and suggests that $\mathrm{Mad} 2$ can remain on weakly attached mouse bivalents, as has been reported in insect spematocytes in meiosis I (Nicklas et al., 2001). However, it is clear that such a weak Mad2 signal is not sufficient to transduce a strong SAC-mediated APC/C inhibition, and it may be that, in oocytes, weak or absent attachment to only a few bivalents is insufficient to generate a 'wait-anaphase' signal.

We conclude that the present findings are highly relevant to the etiology of aneuploidy. The rate at which we observe non-aligned bivalents to undergo non-disjunction here at anaphase onset is compatible with the aneuploidy rate we previously reported for this strain by using chromosome spreading techniques (Lane et al., 2010). Given that our data are consistent with attachment being the primary driver of SAC satisfaction, then this explains the lack of a block to meiosis I when many univalents are present through genetic loss of the recombination gene Mlh1 (Nagaoka et al., 2011). Importantly, it also helps explain why the most commonly observed cause of maternal meiosis I-derived Down Syndrome (trisomy 21 ), prevalent in about $40 \%$ of all cases, is associated with a lack of any recombination between the homologous chromosomes (Lamb et al., 1997; Lamb et al., 1996). In such a scenario, the univalents of chromosome 21 during meiosis I would satisfy the SAC by being able to form monopolar attachments. This is highly reminiscent of MUG cells in mitosis, which are generated by preventing DNA replication and so produce a $\mathrm{M}$ phase with unpaired single chromatids. In these cells, mitosis still occurs but in the absence of any tension being able to develop across sister kinetochores (O'Connell et al., 2008). Finally, our data are relevant to the rise in age-related aneuploidy observed both in human and mouse oocytes. Oocytes spend the majority of their lives arrested at the dictyate stage of prophase I, at which time they have little to no capacity to repair damaged cohesion molecules such as Rec8 and SMC1 $\beta$ (Revenkova et al., 2010; Tachibana-Konwalski et al., 2010). As such, with increasing maternal age the ties holding bivalents together become weaker (Chiang et al., 2010; Lister et al., 2010; Merriman et al., 2012). This decline in cohesion with age will probably exacerbate initial incorrect kinetochore attachments, which do not generate a strong 'wait-anaphase' signal, and so fail to inhibit the $\mathrm{APC} / \mathrm{C}$ in oocytes and may go uncorrected by the time of anaphase-onset leading to mis-segregation.

\section{Acknowledgements}

We thank Rey-Huei Chen (Institute of Molecular Biology, Academia Sinica, Taiwan) for the gift of the anti-Mad2 antibody.

\section{Funding}

This work was funded by grants from the National Health and Medical Research Council Australia [569202] and Australian Research Council [DP120100946] to K.T.J. S.I.R.L. is the recipient of a Northcote graduate scholarship awarded by the Menzies Trust and administered through King's College London. Y.Y. is the recipient of a China Scholarship Council PhD scholarship.

\section{Competing interests statement}

The authors declare no competing financial interests.

\section{Supplementary material}

Supplementary material available online at

http://dev.biologists.org/lookup/suppl/doi:10.1242/dev.077040/-/DC1

\section{References}

Amaro, A. C., Samora, C. P., Holtackers, R., Wang, E., Kingston, I. J., Alonso, M., Lampson, M., McAinsh, A. D. and Meraldi, P. (2010). Molecular control of kinetochore-microtubule dynamics and chromosome oscillations. Nat. Cell Biol. 12, 319-329.

Arooz, T., Yam, C. H., Siu, W. Y., Lau, A., Li, K. K. and Poon, R. Y. (2000). On the concentrations of cyclins and cyclin-dependent kinases in extracts of cultured human cells. Biochemistry 39, 9494-9501.

Bain, J., Plater, L., Elliott, M., Shpiro, N., Hastie, C. J., McLauchlan, H., Klevernic, I., Arthur, J. S., Alessi, D. R. and Cohen, P. (2007). The selectivity of protein kinase inhibitors: a further update. Biochem. J. 408, 297-315.

Brito, D. A. and Rieder, C. L. (2006). Mitotic checkpoint slippage in humans occurs via cyclin B destruction in the presence of an active checkpoint. Curr. Biol. $16,1194-1200$.

Brunet, S., Maria, A. S., Guillaud, P., Dujardin, D., Kubiak, J. Z. and Maro, B. (1999). Kinetochore fibers are not involved in the formation of the first meiotic spindle in mouse oocytes, but control the exit from the first meiotic M phase. J. Cell Biol. 146, 1-12.

Chiang, T., Duncan, F. E., Schindler, K., Schultz, R. M. and Lampson, M. A. (2010). Evidence that weakened centromere cohesion is a leading cause of agerelated aneuploidy in oocytes. Curr. Biol. 20, 1522-1528.

Clute, P. and Pines, J. (1999). Temporal and spatial control of cyclin B1 destruction in metaphase. Nat. Cell Biol. 1, 82-87.

De Antoni, A., Pearson, C. G., Cimini, D., Canman, J. C., Sala, V., Nezi, L., Mapelli, M., Sironi, L., Faretta, M., Salmon, E. D. et al. (2005). The Mad1/Mad2 complex as a template for Mad2 activation in the spindle assembly checkpoint. Curr. Biol. 15, 214-225

DeLuca, J. G., Gall, W. E., Ciferri, C., Cimini, D., Musacchio, A. and Salmon, E. D. (2006). Kinetochore microtubule dynamics and attachment stability are regulated by Hec1. Cell 127, 969-982.

Deng, M. Q. and Shen, S. S. (2000). A specific inhibitor of p34(cdc2)/cyclin B suppresses fertilization-induced calcium oscillations in mouse eggs. Biol. Reprod. 62, 873-878.

Deng, M., Gao, J., Suraneni, P. and Li, R. (2009). Kinetochore-independent chromosome poleward movement during anaphase of meiosis II in mouse eggs. PLOS ONE 4, e5249.

Dumont, J., Oegema, K. and Desai, A. (2010). A kinetochore-independent mechanism drives anaphase chromosome separation during acentrosomal meiosis. Nat. Cell Biol. 12, 894-901.

Gassmann, R., Holland, A. J., Varma, D., Wan, X., Civril, F., Cleveland, D. W., Oegema, K., Salmon, E. D. and Desai, A. (2010). Removal of Spindly from microtubule-attached kinetochores controls spindle checkpoint silencing in human cells. Genes Dev. 24, 957-971.

Gavet, O. and Pines, J. (2010). Progressive activation of CyclinB1-Cdk1 coordinates entry to mitosis. Dev. Cell 18, 533-543.

Gorr, I. H., Reis, A., Boos, D., Wuhr, M., Madgwick, S., Jones, K. T. and Stemmann, O. (2006). Essential CDK1-inhibitory role for separase during meiosis I in vertebrate oocytes. Nat. Cell Biol. 8, 1035-1037.

Griffis, E. R., Stuurman, N. and Vale, R. D. (2007). Spindly, a novel protein essential for silencing the spindle assembly checkpoint, recruits dynein to the kinetochore. J. Cell Biol. 177, 1005-1015.

Hached, K., Xie, S. Z., Buffin, E., Cladiere, D., Rachez, C., Sacras, M., Sorger, P. K. and Wassmann, K. (2011). Mps1 at kinetochores is essential for female mouse meiosis I. Development 138, 2261-2271.

Hassold, T. and Hunt, P. (2009). Maternal age and chromosomally abnormal pregnancies: what we know and what we wish we knew. Curr. Opin. Pediatr. 21, 703-708.

Herbert, M., Levasseur, M., Homer, H., Yallop, K., Murdoch, A. and McDougall, A. (2003). Homologue disjunction in mouse oocytes requires proteolysis of securin and cyclin B1. Nat. Cell Biol. 5, 1023-1025.

Holland, A. J. and Cleveland, D. W. (2009). Boveri revisited: chromosomal instability, aneuploidy and tumorigenesis. Nat. Rev. Mol. Cell Biol. 10, 478-487.

Holt, J. E., Weaver, J. and Jones, K. T. (2010). Spatial regulation of APCCdh1induced cyclin B1 degradation maintains $\mathrm{G} 2$ arrest in mouse oocytes. Development 137, 1297-1304.

Holt, J. E., Tran, S. M., Stewart, J. L., Minahan, K., Garcia-Higuera, I., Moreno, S. and Jones, K. T. (2011). The APC/C activator FZR1 coordinates the timing of meiotic resumption during prophase I arrest in mammalian oocytes. Development 138, 905-913.

Homer, H. A., McDougall, A., Levasseur, M., Yallop, K., Murdoch, A. P. and Herbert, M. (2005). Mad2 prevents aneuploidy and premature proteolysis of cyclin B and securin during meiosis I in mouse oocytes. Genes Dev. 19, 202-207.

Hornick, J. E., Mader, C. C., Tribble, E. K., Bagne, C. C., Vaughan, K. T., Shaw, S. L. and Hinchcliffe, E. H. (2011). Amphiastral mitotic spindle assembly in vertebrate cells lacking centrosomes. Curr. Biol. 21, 598-605.

Jaqaman, K., King, E. M., Amaro, A. C., Winter, J. R., Dorn, J. F., Elliott, H. L., McHedlishvili, N., McClelland, S. E., Porter, I. M., Posch, M. et al. (2010). Kinetochore alignment within the metaphase plate is regulated by centromere stiffness and microtubule depolymerases. J. Cell Biol. 188, 665-679.

Jin, F., Hamada, M., Malureanu, L., Jeganathan, K. B., Zhou, W., Morbeck, D. E. and van Deursen, J. M. (2010). Cdc20 is critical for meiosis I and fertility of female mice. PLoS Genet. 6, e1001147. 
Jones, K. T. (2008). Meiosis in oocytes: predisposition to aneuploidy and its increased incidence with age. Hum. Reprod. Update 14, 143-158.

Kanatsu-Shinohara, M., Schultz, R. M. and Kopf, G. S. (2000). Acquisition of meiotic competence in mouse oocytes: absolute amounts of p34(cdc2), cyclin B1, cdc25C, and wee1 in meiotically incompetent and competent oocytes. Biol. Reprod. 63, 1610-1616.

Khodjakov, A. and Pines, J. (2010). Centromere tension: a divisive issue. Nat. Cell Biol. 12, 919-923.

Kim, S., Sun, H., Ball, H. L., Wassmann, K., Luo, X. and Yu, H. (2010) Phosphorylation of the spindle checkpoint protein Mad2 regulates its conformational transition. Proc. Natl. Acad. Sci. USA 107, 19772-19777.

Kitajima, T. S., Ohsugi, M. and Ellenberg, J. (2011). Complete kinetochore tracking reveals error-prone homologous chromosome biorientation in mammalian oocytes. Cell 146, 568-581.

Kouznetsova, A., Lister, L., Nordenskjold, M., Herbert, M. and Hoog, C. (2007). Bi-orientation of achiasmatic chromosomes in meiosis I oocytes contributes to aneuploidy in mice. Nat. Genet. 39, 966-968.

Kulukian, A., Han, J. S. and Cleveland, D. W. (2009). Unattached kinetochores catalyze production of an anaphase inhibitor that requires a Mad2 template to prime Cdc20 for BubR1 binding. Dev. Cell 16, 105-117.

Lamb, N. E., Freeman, S. B., Savage-Austin, A., Pettay, D., Taft, L., Hersey, J., Gu, Y., Shen, J., Saker, D., May, K. M. et al. (1996). Susceptible chiasmate configurations of chromosome 21 predispose to non-disjunction in both maternal meiosis I and meiosis II. Nat. Genet. 14, 400-405

Lamb, N. E., Feingold, E., Savage, A., Avramopoulos, D., Freeman, S., Gu, Y., Hallberg, A., Hersey, J., Karadima, G., Pettay, D. et al. (1997). Characterization of susceptible chiasma configurations that increase the risk for maternal nondisjunction of chromosome 21. Hum. Mol. Genet. 6, 1391-1399.

Lane, S. I., Chang, H. Y., Jennings, P. C. and Jones, K. T. (2010). The Aurora kinase inhibitor ZM447439 accelerates first meiosis in mouse oocytes by overriding the spindle assembly checkpoint. Reproduction 140, 521-530.

Ledan, E., Polanski, Z., Terret, M. E. and Maro, B. (2001). Meiotic maturation of the mouse oocyte requires an equilibrium between cyclin $B$ synthesis and degradation. Dev. Biol. 232, 400-413.

LeMaire-Adkins, R., Radke, K. and Hunt, P. A. (1997). Lack of checkpoint control at the metaphase/anaphase transition: a mechanism of meiotic nondisjunction in mammalian females. J. Cell Biol. 139, 1611-1619.

Li, M., York, J. P. and Zhang, P. (2007). Loss of Cdc20 causes a securindependent metaphase arrest in two-cell mouse embryos. Mol. Cell. Biol. 27, 3481-3488.

Li, M., Li, S., Yuan, J., Wang, Z. B., Sun, S. C., Schatten, H. and Sun, Q. Y. (2009). Bub3 is a spindle assembly checkpoint protein regulating chromosome segregation during mouse oocyte meiosis. PLOS ONE 4, e7701.

Lister, L. M., Kouznetsova, A., Hyslop, L. A., Kalleas, D., Pace, S. L., Barel, J. C., Nathan, A., Floros, V., Adelfalk, C., Watanabe, Y. et al. (2010). Agerelated meiotic segregation errors in mammalian oocytes are preceded by depletion of cohesin and Sgo2. Curr. Biol. 20, 1511-1521.

Liu, D., Vader, G., Vromans, M. J., Lampson, M. A. and Lens, S. M. (2009). Sensing chromosome bi-orientation by spatial separation of aurora B kinase from kinetochore substrates. Science 323, 1350-1353.

Madgwick, S., Nixon, V. L., Chang, H. Y., Herbert, M., Levasseur, M. and Jones, K. T. (2004). Maintenance of sister chromatid attachment in mouse eggs through maturation-promoting factor activity. Dev. Biol. 275, 68-81.

Madgwick, S., Hansen, D. V., Levasseur, M., Jackson, P. K. and Jones, K. T. (2006). Mouse Emi2 is required to enter meiosis II by reestablishing cyclin B1 during interkinesis. J. Cell Biol. 174, 791-801.

Mahoney, N. M., Goshima, G., Douglass, A. D. and Vale, R. D. (2006). Making microtubules and mitotic spindles in cells without functional centrosomes. Curr. Biol. 16, 564-569.

Maresca, T. J. and Salmon, E. D. (2010). Welcome to a new kind of tension: translating kinetochore mechanics into a wait-anaphase signal. J. Cell Sci. 123, 825-835.

McGuinness, B. E., Anger, M., Kouznetsova, A., Gil-Bernabe, A. M., Helmhart, W., Kudo, N. R., Wuensche, A., Taylor, S., Hoog, C., Novak, B. et al. (2009). Regulation of APC/C activity in oocytes by a Bub1-dependent spindle assembly checkpoint. Curr. Biol. 19, 369-380

Merriman, J., Jennings, P., McLaughlin, E. and Jones, K. T. (2012). Effect of aging on superovulation efficiency, aneuploidy rates, and sister chromatid cohesion in mice aged up to 15-months. Biol. Reprod. 86, 49.

Musacchio, A. and Salmon, E. D. (2007). The spindle-assembly checkpoint in space and time. Nat. Rev. Mol. Cell Biol. 8, 379-393.

Nagaoka, S. I., Hodges, C. A., Albertini, D. F. and Hunt, P. A. (2011). Oocytespecific differences in cell-cycle control create an innate susceptibility to meiotic errors. Curr. Biol. 21, 651-657.

Nicklas, R. B., Waters, J. C., Salmon, E. D. and Ward, S. C. (2001). Checkpoint signals in grasshopper meiosis are sensitive to microtubule attachment, but tension is still essential. J. Cell Sci. 114, 4173-4183.

O'Connell, C. B., Loncarek, J., Hergert, P., Kourtidis, A., Conklin, D. S. and Khodjakov, A. (2008). The spindle assembly checkpoint is satisfied in the absence of interkinetochore tension during mitosis with unreplicated genomes J. Cell Biol. 183, 29-36.

Peters, J. M. (2006). The anaphase promoting complex/cyclosome: a machine designed to destroy. Nat. Rev. Mol. Cell Biol. 7, 644-656.

Polanski, Z., Ledan, E., Brunet, S., Louvet, S., Verlhac, M. H., Kubiak, J. Z. and Maro, B. (1998). Cyclin synthesis controls the progression of meiotic maturation in mouse oocytes. Development 125, 4989-4997.

Potapova, T. A., Daum, J. R., Pittman, B. D., Hudson, J. R., Jones, T. N., Satinover, D. L., Stukenberg, P. T. and Gorbsky, G. J. (2006). The reversibility of mitotic exit in vertebrate cells. Nature $\mathbf{4 4 0}, 954-958$.

Reis, A., Chang, H. Y., Levasseur, M. and Jones, K. T. (2006). APCcdh1 activity in mouse oocytes prevents entry into the first meiotic division. Nat. Cell Biol. 8 539-540.

Reis, A., Madgwick, S., Chang, H. Y., Nabti, I., Levasseur, M. and Jones, K. T. (2007). Prometaphase APCcdh1 activity prevents non-disjunction in mammalian oocytes. Nat. Cell Biol. 9, 1192-1198.

Revenkova, E., Herrmann, K., Adelfalk, C. and Jessberger, R. (2010). Oocyte cohesin expression restricted to predictyate stages provides full fertility and prevents aneuploidy. Curr. Biol. 20, 1529-1533.

Rieder, C. L., Cole, R. W., Khodjakov, A. and Sluder, G. (1995). The checkpoint delaying anaphase in response to chromosome monoorientation is mediated by an inhibitory signal produced by unattached kinetochores. J. Cell Biol. 130, 941948.

Sakuno, T., Tanaka, K., Hauf, S. and Watanabe, Y. (2011). Repositioning of aurora B promoted by chiasmata ensures sister chromatid mono-orientation in meiosis I. Dev. Cell 21, 534-545.

Salmon, E. D. and Segall, R. R. (1980). Calcium-labile mitotic spindles isolated from sea urchin eggs (Lytechinus variegatus). J. Cell Biol. 86, 355-365.

Santaguida, S. and Musacchio, A. (2009). The life and miracles of kinetochores. $E M B O$ J. 28, 2511-2531.

Schindler, K. and Schultz, R. M. (2009). The CDC14A phosphatase regulates oocyte maturation in mouse. Cell Cycle 8, 1090-1098.

Schmitz, M. H., Held, M., Janssens, V., Hutchins, J. R., Hudecz, O., Ivanova, E., Goris, J., Trinkle-Mulcahy, L., Lamond, A. I., Poser, I. et al. (2010). Livecell imaging RNAi screen identifies PP2A-B55alpha and importin-beta1 as key mitotic exit regulators in human cells. Nat. Cell Biol. 12, 886-893.

Skibbens, R. V., Skeen, V. P. and Salmon, E. D. (1993). Directional instability of kinetochore motility during chromosome congression and segregation in mitotic newt lung cells: a push-pull mechanism. J. Cell Biol. 122, 859-875.

Tachibana-Konwalski, K., Godwin, J., van der Weyden, L., Champion, L., Kudo, N. R., Adams, D. J. and Nasmyth, K. (2010). Rec8-containing cohesin maintains bivalents without turnover during the growing phase of mouse oocytes. Genes Dev. 24, 2505-2516.

Thompson, S. L. and Compton, D. A. (2008). Examining the link between chromosomal instability and aneuploidy in human cells. J. Cell Biol. 180, 665-672.

Toso, A., Winter, J. R., Garrod, A. J., Amaro, A. C., Meraldi, P. and McAinsh, A. D. (2009). Kinetochore-generated pushing forces separate centrosomes during bipolar spindle assembly. J. Cell Biol. 184, 365-372.

Verlhac, M. H., Lefebvre, C., Guillaud, P., Rassinier, P. and Maro, B. (2000) Asymmetric division in mouse oocytes: with or without Mos. Curr. Biol. 10, 1303-1306.

Wassmann, K., Niault, T. and Maro, B. (2003). Metaphase I arrest upon activation of the Mad2-dependent spindle checkpoint in mouse oocytes. Curr. Biol. 13, 1596-1608.

Wei, L., Liang, X. W., Zhang, Q. H., Li, M., Yuan, J., Li, S., Sun, S. C., Ouyang, Y. C., Schatten, H. and Sun, Q. Y. (2010). BubR1 is a spindle assembly checkpoint protein regulating meiotic cell cycle progression of mouse oocyte. Cell Cycle 9, 1112-1121

Weisenberg, R. C. and Deery, W. J. (1981). The mechanism of calcium-induced microtubule disassembly. Biochem. Biophys. Res. Commun. 102, 924-931.

Welburn, J. P., Vleugel, M., Liu, D., Yates, J. R., 3rd, Lampson, M. A., Fukagawa, T. and Cheeseman, I. M. (2010). Aurora B phosphorylates spatially distinct targets to differentially regulate the kinetochore-microtubule interface. Mol. Cell 38, 383-392

Williams, B. R. and Amon, A. (2009). Aneuploidy: cancer's fatal flaw? Cancer Res. 69, 5289-5291.

Yang, M., Li, B., Tomchick, D. R., Machius, M., Rizo, J., Yu, H. and Luo, X. (2007). p31comet blocks Mad2 activation through structural mimicry. Cell 131, 744-755.

Yang, K. T., Li, S. K., Chang, C. C., Tang, C. J., Lin, Y. N., Lee, S. C. and Tang, T. K. (2010). Aurora-C kinase deficiency causes cytokinesis failure in meiosis I and production of large polyploid oocytes in mice. Mol. Biol. Cell 21, 2371-2383.

Yin, S., Wang, Q., Liu, J. H., Ai, J. S., Liang, C. G., Hou, Y., Chen, D. Y. Schatten, H. and Sun, Q. Y. (2006). Bub1 prevents chromosome misalignment and precocious anaphase during mouse oocyte meiosis. Cell Cycle 5, 2130-2137.

$\mathbf{Y u}, \mathbf{H}$. (2006). Structural activation of Mad2 in the mitotic spindle checkpoint: the two-state Mad2 model versus the Mad2 template model. J. Cell Biol. 173, 153157

Yu, H. (2007). Cdc20: a WD40 activator for a cell cycle degradation machine. Mol. Cell 27, 3-16. 